\title{
Cloning and Enzymatic Activity Analysis of the Malonyl-CoA:acyl Carrier Protein Transacylase in Brassica napus Cultivars with Different Oil Content
}

\author{
Chenning Qu1 ${ }^{1}$ Huanhuan Jiang ${ }^{2}$, Lei Zhang1* \\ ${ }^{1}$ State Key Laboratory of Hybrid Rice, Wuhan University, Wuhan, China \\ ${ }^{2}$ BGI-Shenzhen, Shenzhen, China \\ Email: ${ }^{*}$ arabilab@whu.edu.cn
}

Received 18 May 2014; revised 28 June 2014; accepted 24 July 2014

Copyright (C) 2014 by authors and Scientific Research Publishing Inc.

This work is licensed under the Creative Commons Attribution International License (CC BY). http://creativecommons.org/licenses/by/4.0/

(c) $\underset{\mathrm{EY}}{(1)}$ Open Access

\section{Abstract}

The full-length genomic DNA of MCAT (Malonyl-CoA:acyl carrier protein transacylase) in Brassica napus was cloned. BnMCAT shares very high identity with AtMCAT in gene sequence and gene structure. A multiple alignment of the protein sequence showed that BnMCAT shares high identity with other MCATs from E. coli and plants. BnMCAT was expressed in all tissues, such as roots, stems, leaves, flowers, and seeds, and no significant differences in the expression level were found in different embryo stages after pollination. According to an in vitro relative activity analysis, purified recombinant BnMCAT expressed in E. coli had transacylase activity. Although the relative activities of BnMCAT in crude extracts isolated from different staged embryos were similar and showed little variation, a higher relative activity was found in a crude extract isolated from embryos in comparison to leaves. Different relative activities of BnMCAT in crude extracts isolated from cultivars with different oil content were also found, suggesting that the activity of BnMCAT might be a decisive factor for a high oil content. Together, these results showed that BnMCAT is an important enzyme in the FAS system and indicate that BnMCAT might be a new target enzyme for future crop improvement through genetic engineering.

\section{Keywords}

Malonyl-CoA:acyl Carrier Protein Transacylase (MCAT), Brassica Napus, Oil Content, Relative Activities

\footnotetext{
${ }^{*}$ Corresponding author.
} 


\section{Introduction}

Fatty acid biosynthesis is catalyzed by different enzymes, depending on the species. These enzymes can be divided into two types. In the type I (Fatty acid synthesis I, FAS I) system found in many eukaryotes, the biosynthesis of fatty acids is catalyzed by a single large and multifunctional polypeptide. In contrast, in the type II (Fatty acid synthesis II, FAS II) system found in most bacteria and plants, the biosynthesis of fatty acids is catalyzed by a series of small soluble proteins that are each encoded by discrete genes [1].

The malonyl-CoA:acyl carrier protein transacylase (MCAT), a key enzyme in the FAS II pathway, is responsible for transferring the malonyl group from malonyl-CoA (malonyl-coenzyme A) to the -SH group of ACP (acyl carrier protein) and then forming the free CoASH and malonyl-ACP. Malonyl-ACP is the two-carbon donor required for the elongation steps in fatty acid biosynthesis. Previous studies indicate that the MCAT enzyme performs a two-step reaction in which MCAT first binds to malonyl-CoA to form a stable tetrahedral malonyl-MCAT intermediate; the holo-ACP then docks on the surface of the intermediate via a hydrophobic interaction to receive the malonyl chain. In fact, MCAT induces an upstream reaction and may be the essential building block in the fatty acid biosynthesis cycle [2] [3]. In addition, MCAT can provide acyl-ACP thioesters for the biosynthesis of aromatic polyketides, including the largest classes of secondary metabolites, such as tetracyclines and erythromycins [3] [4].

A partial cDNA of the MCAT gene was previously cloned from Brassica napus; this sequence encodes 351 amino acids and shows $47 \%$ homology to E. coli MCAT. The partial B. napus MCAT clone can restore the growth of a temperature-sensitive E. coli MCAT mutant (Fab D89) at $39^{\circ} \mathrm{C}$ [5]. However, no full-length cDNA or genomic DNA of Brassica napus MCAT has been reported to date.

Oils are glycerol triesters of fatty acids and are mainly derived from plant sources. Brassica napus, a widely grown plant, is an important source of edible oil, providing approximately $13 \%$ of the world's supply of vegetable oil [6]. Thus, it is important to study the fatty acid biosynthesis pathway to improve oil quality and increase the oil content of Brassica napus. Indeed, investigations of MCAT, an important enzyme in the FAS II system, will help to elucidate the basis molecular mechanism of fatty acid biosynthesis in Brassica napus.

In this study, we report the full-length genomic DNA and full-length open reading frame of MCAT in Brassica napus. BnMCAT shares a very high identity with AtMCAT in gene sequence and gene structure. A multiple alignment of the protein sequence showed that BnMCAT also shares high identity with other MCATs from $E$. coli and plants. BnMCAT was found to be expressed in all tissues, including the root, stem, leaf, flower, and seed, and no significant differences in expression were found in embryos of different stages after pollination. An in vitro relative activity analysis showed that purified recombinant $E$. coli-expressed BnMCAT possesses transacylase activity. The relative activities of BnMCAT in crude extracts isolated from embryos at different stages were similar, with little variation. However, a higher relative activity was found in an embryo crude extract than leaf crude extract. Different relative activities of BnMCAT in crude extracts of cultivars with different oil content were also found, suggesting that the activity of BnMCAT might be a decisive factor in a high oil content. All these results demonstrate that BnMCAT is an important enzyme in the FAS system and indicate that BnMCAT might be a new target enzyme in future crop improvement through genetic engineering.

\section{Materials and Methods}

\subsection{RNA Isolation}

Brassica napus was grown in the farm of Nanjing Agricultural University and the greenhouse of Wuhan University. Immature seeds at 20 - 40 days after pollination (DAP) were collected, immediately frozen in liquid N2, and stored in a freezer at $-80^{\circ} \mathrm{C}$. Total RNA was extracted from the immature seeds using Plant RNA Purification Reagent (Invitrogen). When necessary, the total RNA was treated with RNase-free DNase I (ToYoBo). The first-strand cDNA was synthesized using $2 \mu \mathrm{g}$ of total RNA with Superscript II (Invitrogen) and Oligo(dT)18.

\subsection{RT-PCR and Gene Cloning}

The following primers were designed based on the partial sequence of BnMCAT cDNA published by Simon and Slabas [5]: BnMCAT 5' primer 1 (5' TTGTTCGCCGACTACAAACCC 3') and BnMCAT 3' primer 1 (5' GATGTTTTCGACGCTAGCA 3'). PCR was conducted in a 50- $\mu$ l final volume including $0.5 \mu \mathrm{l}$ cDNA tem- 
plate, $1 \times$ LA Taqase buffer, $200 \mu \mathrm{M}$ deoxynucleotide triphosphates (dNTPs), $400 \mathrm{nM}$ each primer, and 2 units LA Taqase polymerase (TaKaRa). The PCR conditions included an initial denaturation at $94^{\circ} \mathrm{C}$ for 3 min, followed by 30 cycles of $94^{\circ} \mathrm{C}$ for $30 \mathrm{~s}, 55^{\circ} \mathrm{C}$ for $30 \mathrm{~s}$, and $72^{\circ} \mathrm{C}$ for 1 min per kb, with a final extension at $72^{\circ} \mathrm{C}$ for $10 \mathrm{~min}$. The PCR products were gel purified using a quick gel extraction kit, cloned into the pBS-T vector, and sequenced from both ends by Sunny Biology Company. BnMCAT 5' primer 2 (5' ATGCGTTCACTGCTTCACCG 3') and BnMCAT 3' primer 2 (5' CCGCCTCACGAACACGG 3') were designed based on the sequencing of the PCR product and the AtMCAT CDNA sequence. The PCR conditions were the same as described above.

For the semi-quantitative PCR analysis, $0.5 \mu \mathrm{l}$ of the reverse transcription reaction was subjected to PCR using gene-specific primers, BnMCAT 5' primer 1 (5' TTGTTCGCCGACTACAAACCC 3') and BnMCAT 3' primer 1 (5' GATGTTTTCGACGCTAGCA 3'). Constitutively expressed Ubiquitin or Actin was used as an internal control. Each PCR assay was independently performed at least three times.

\subsection{Cloning of Genomic BnMCAT DNA}

The genomic DNA of Brassica napus was isolated using the CTAB method. The BnMCAT genomic DNA was amplified by PCR using BnMCAT 5' primer 2 (5' ATGCGTTCACTGCTTCACCG 3') and BnMCAT 3' primer 1 (5' GATGTTTTCGACGCTAGCA 3'). The PCR product was sequenced by Sunny Biology Company.

\subsection{Alignment of the MCATs from Different Cultivars}

Omiga software was used to align the MCATs from different species of $B$. napus. BLAST at NCBI web (http://blast.ncbi.nlm.nih.gov/Blast.cgi) was used to analyze the multiple sequence alignments of MCATs isolated from plants, animals, and pathogens. Sequence reassembly was performed using the DNAStar software (http://www.DNAStar.com).

\subsection{Expression and Purification of BnMCAT in $E_{\text {. coli }}$}

The BnMCAT gene was amplified by PCR from Brassica napus cDNA using the following primers: BnMCATsense, 5'-CG GAA TTC ATG CGT TCA CTG CTT CAC-3' (containing an EcoR I restriction site, underlined) and BnMCAT-antisense, 5'-CCG CTC GAG TCA AGC ACT GAT GTT TTC-3' (containing an Xho I restriction site, underlined). The 1196-base pair PCR product was purified, digested with EcoR I and Xho I, and ligated to pET28a (Novagen) digested with the same enzymes. The DNA insert was sequenced to verify the absence of PCR artifacts. The resulting plasmid, designated pET28a::BnMCAT, was used to transform E. coli DE3 (BL21) for over-expression of the recombinant protein.

An overnight culture of $E$. coli DE3 (BL21) carrying pET28a::BnMCAT was used to inoculate a large volume of LB broth supplemented with $25 \mu \mathrm{g} / \mathrm{ml}$ kanamycin and incubated at $37^{\circ} \mathrm{C}$ with shaking until the optical density at $600 \mathrm{~nm}$ reached $0.6-0.8$. The culture was then induced with $1 \mathrm{mM}$ isopropyl- $\beta$-D-thiogalactopyranoside (IPTG). Growth was continued for another $4 \mathrm{~h}$ at $37^{\circ} \mathrm{C}$, and the cells were harvested by centrifugation. The pellet was resuspended in breaking buffer ( $20 \mathrm{mM}$ phosphate buffer (pH 7.4), $0.5 \mathrm{M} \mathrm{NaCl}, 0.1 \mathrm{mg} / \mathrm{ml}$ lysozyme (Sigma), with DNase, RNase, and $1 \mathrm{mM}$ PMSF) on ice for approximately $30 \mathrm{~min}$. After brief sonication, the mixture was centrifuged at $14,000 \mathrm{r} / \mathrm{min}$ for $30 \mathrm{~min}$ at $4^{\circ} \mathrm{C}$. The supernatant was collected and applied to an Ni-NTA column ( $1 \mathrm{ml}$, QIAGEN) that had been equilibrated with breaking buffer. The column was extensively washed with phosphate buffer ( $\mathrm{pH} 7.4) / 0.5 \mathrm{M} \mathrm{NaCl}$ and eluted with a stepwise gradient of imidazole (50 mM $200 \mathrm{mM})$. Fractions $(1 \mathrm{ml})$ were collected, and the presence of BnMCAT was detected by 12\% SDS-PAGE. Fractions containing pure BnMCAT were pooled, dialyzed against $20 \mathrm{mM}$ Tris/ $\mathrm{HCl}$ (pH 7.4), and used for enzyme analyses or stored at $-70^{\circ} \mathrm{C}$.

\subsection{Assay of Malonyl-CoA:ACP Transacylase in Vitro}

Immature seeds at 20 - 40 days after pollination (DAP) were peeled and ground in extraction buffer containing $20 \mathrm{mM}$ Tris-HCl (pH 8.0), $2 \mathrm{mM}$ dithiothreitol, and 5\% (v/v) glycerol at $100 \mathrm{mg}$ of tissue per ml of buffer. The initial homogenization and all subsequent steps were performed at $4^{\circ} \mathrm{C}$. The homogenates were centrifuged at $8000 \mathrm{r} / \mathrm{min}$ for $30 \mathrm{~min}$ to remove the debris, and the pellets were redissolved and dialyzed against $25 \mathrm{mM}$ Tris buffer with $\mathrm{pH} 7.8$ for $6 \mathrm{~h}$. The dialyzed fraction as the crude extract was immediately used to analyze the en- 
zymatic activity of MCAT.

The activity of malonyl-CoA:ACP transacylase was assayed in a final volume of $150 \mu \mathrm{l}$ with $120 \mu \mathrm{l}$ of crude extract using a modification of the method described by Martínez-Force et al. [7] and Kremer et al. [8]. The crude extract was omitted from control assays. Reaction mixtures containing $20 \mathrm{mM}$ Tris- $\mathrm{HCl}(\mathrm{pH}$ 8.0), $17 \mu \mathrm{M}$ ACP (E. coli FAS holo-ACP, purchased from Sigma), $5 \mu \mathrm{M}\left[2-{ }^{14} \mathrm{C}\right]$ malonyl-CoA $(4.5 \mathrm{mCi} / \mathrm{mmol})$, and $0.5 \mathrm{mM}$ 2-mercaptoethanol were incubated for $1 \mathrm{~min}$ at room temperature. The reactions were stopped by the addition of the same volume of $10 \%$ trichloroacetic acid (TCA), maintaining the mixture on ice for $10 \mathrm{~min}$. The samples were diluted with $1 \mathrm{ml}$ of 5\% TCA and centrifuged ( $5 \mathrm{~min}$ at 15,000 $\times \mathrm{g}$ ). The supernatants were discarded, and the pellets were washed with $1 \mathrm{ml}$ of 5\% TCA and then re-dissolved in $25 \mu \mathrm{l}$ of $50 \mathrm{mM} \mathrm{MES} \mathrm{(pH} \mathrm{6.3).} \mathrm{After}$ adding $3 \mathrm{ml}$ of solvent scintillant reagent, the radio-activity was measured using a calibrated scintillation counter (Beckman LS 6500). EXCEL software were used in statistics analysis. Data are means ( \pm SD) of three biological replicates.

The protein concentration was determined by the method of Bradford using BSA as a standard [9].

\section{Results}

\subsection{Cloning and Sequence Analysis of BnMCAT}

MCAT is a key enzyme in the plant fatty acid biosynthesis pathway, and MCAT cDNA sequences have been isolated from a number of plants, such as Arabidopsis thaliana, Perilla frutescens, Capsicum annuum, Oryza sativa, and Arachis hypogaea. The partial cDNA of BnMCAT without the start codon was isolated in 1998 by Simon and Slabas [5], and full-length BnMCAT was cloned based on this result. The full-length BnMCAT sequence contains an OFR of $1179 \mathrm{bp}$, with a start codon ATG and a stop codon TGA, and shows very high similarity in both sequence and structure with AtMCAT (76\% identity in genomic DNA sequence and 89\% identity in OFR sequence) (Figure 1(a) and Figure 1(b)).

The partial cDNA of BnMCAT reported by Simon and Slabas [5] has 1200 bp, encoding 351 amino acids that show $47 \%$ homology to E. coli MCAT [5]. The deduced amino acid sequence of full-length BnMCAT consists of 392 residues, with a calculated molecular mass of $41.5 \mathrm{kDa}$ and a $P I$ of 8.88 . Although the partial BnMCAT sequence shows $47 \%$ homology to E. coli MCAT and can functionally complement the E. coli mutant Fad D89 [5], the full-length gene does not show high homology (only 33\%) with E. coli MCAT. In contrast, the full-length BnMCAT shares high homology with other MCAT sequences isolated from plants, such as AtMCAT (88\%), OsMCAT (71\%), AhMCAT (77\%), CaMCAT (74\%), GmMCAT (84\%), and PfMCAT (73\%) (Figure 2).

The following was previously reported for the E. coli MCAT active site: Ser92 is hydrogen bonded to His201, Gln250 can serve as an H-bond acceptor, Gln11 can serve as an H-bond donor during interaction with His201, and Arg117 might play a role in binding the free carboxyl group [6] [10]. BnMCAT, Gln96, Ser181, Arg206, His294, and Gln343, equivalent to E. coli MCAT Gln11, Ser92, Arg117, His201, and Gln250, are conserved in higher plants, including Arabidopsis thaliana, Oryza sativa, Arachis hypogaea, Capsicum annuum, Glycine max, and Perilla frutescens (Figure 2). The GLSLGEY motif containing the catalytic residue is completely conserved in higher plants and is comparable to the GHSLGEY motif in $E$. coli MCAT (Figure 2).

\subsection{The Expression of BnMCAT in Brassica napus}

RT-PCR was used to analyze the expression of BnMCAT in Brassica napus. BnMCAT was expressed in all the tissues, including roots, stems, leaves, flowers, and seeds (Figure 3(a)). MCAT is a key enzyme in the FAS II pathway to synthesize the fatty acids that are the important components of lipids, which are the basic components of the cellular membranes [11]. Accordingly, it is necessary for MCAT to be expressed in all tissues to provide these necessary fatty acids as material for lipid synthesis.

As the site of rapeseed oil storage, a large amount of fatty acids are biosynthesized in the seed. Therefore, the expression level of BnMCAT at different stages of the immature seed was also analyzed (Figure 3(b)). No significant differences in the expression level were found in different embryo stages at 10, 20, 30, 35, and 40 days after pollination, suggesting that MCAT might be sufficient to meet the normal fatty acid synthesis demand in plants [12]. 


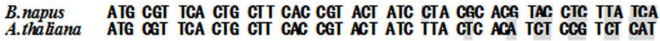

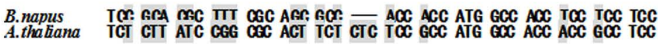

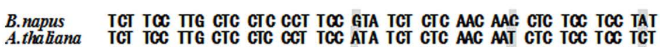
B.napus
A.thriana AGA AAT $\odot C C$ TCC TCC CTC GCA TIC TCC GTC AAG AAT CTC ACC CGA TCC

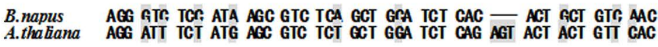

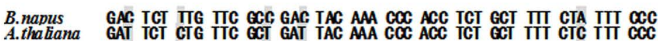

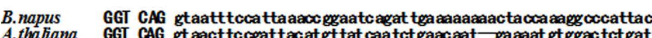

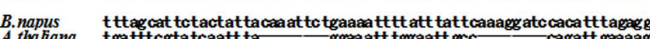

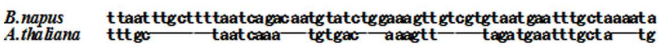

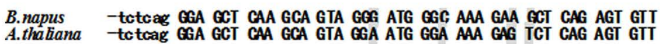

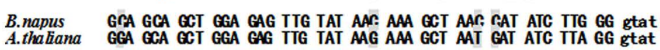

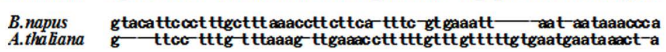
B.napus
A.thnana B.napus
A.thriana

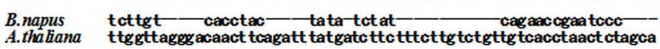
B.napus - tttttatgt tetttagetttatt- - -

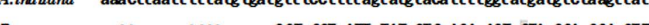

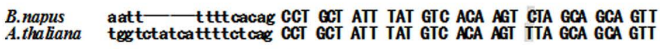
B.npopus
A.thriana
GAA TTG CTT OGI GTC CGT GA GGC GGG GAG CAG ATA ATT AAC TCC GTC B.napus
A.thanana GAT GTC ACT TGT @GT CTC AGC TTG GGA GAG TAC ACT GCT CTC GCA ITT

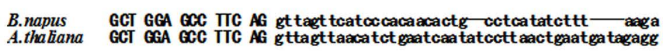

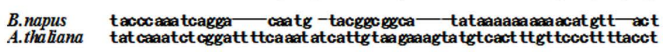

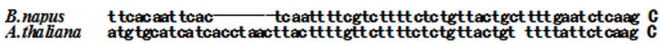

B.napus IIT GAG GAT GGG CIA AAG CII GIA AM CIC AGA GGA GA GCG ATG CAG
A.than

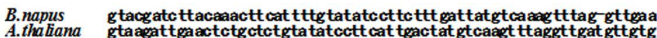

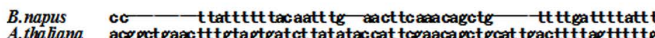

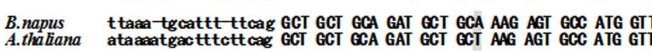

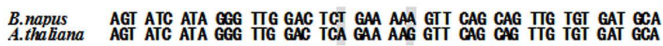
B.napus
A.thinama GCA AAT CAA GAA GTA GAA GA GCT GAC AAA GIT CAG ATC GCT AAT TTC

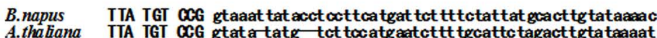
B.napus cattttcacattocatta-cactt totgtt tacagBST AAC TAC OCA GTA TCI GGA

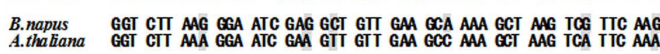

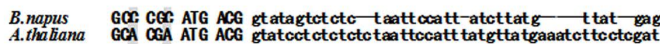

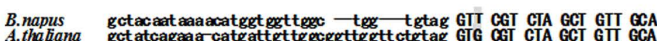

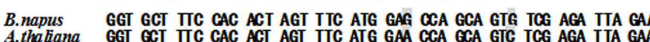
B.napus GCT GCA TGG GCA TCA ACG GAG ATC AGA AGI CCG AGG ATC CCA GTG ATC

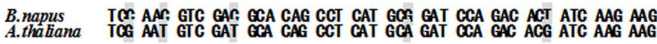

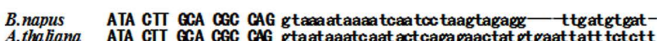
B.nppus B.napus CCA GTG GAA TGG GAG ACA ACG GTG AAG ACT CTC TTA TTC AAA GGG GT

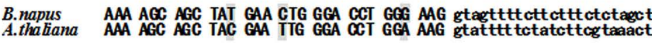

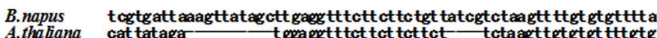

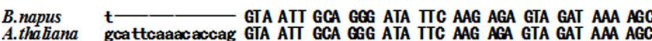
B.napus GCA AGT TIC GA AAC ATC AGT GCT TEA A.thatiana GCA AGI TTC GAA AAC ATC AGT GCT TGA

(a)

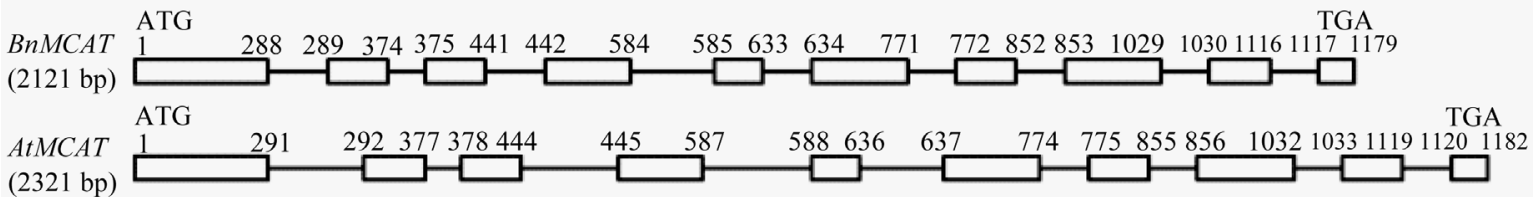

(b)

Figure 1. Sequence and structure analyses of BnMCAT. (a) Nucleotide sequence of BnMCAT and AtMCAT. The DNA sequences were aligned using the OMIGA software, with some modification based on codon usage; (b) The structure of BnMCAT and AtMCAT genomic DNA.

\subsection{Expression and Purification of BnMCAT in E. coli and Enzymatic Activity Analysis}

Three BnMCAT genes from three cultivars with different oil content, named 7352, 7405, and 7708, were over-expressed in E. coli. The recombinant proteins were purified by affinity chromatography using a $\mathrm{Ni}^{+}-\mathrm{NTA}^{-}$ affinity column. The SDS-PAGE results showed that pure recombinant proteins were eluted with $100 \mathrm{mM}$ imidazole (Figure 4). After dialysis against $10 \mathrm{mM}$ Tris/ $\mathrm{HCl}\left(\mathrm{pH} \mathrm{7.4)}\right.$ for approximately $8 \mathrm{~h}$ at $4^{\circ} \mathrm{C}$, each recombinant BnMCAT was used for an enzymatic activity analysis.

All the recombinant proteins showed high transacylase activity in an in vitro enzymatic activity assay, with no difference in enzymatic activity found among the three recombinant proteins derived from cultivars with different oil content (Table 1). This result indicated that, although there is some variation in amino acid sequence among these BnMCATs, these amino acids might not be key residues for enzymatic activity.

The kinetic parameters of the three recombinant BnMCATs were determined using double-reciprocal analyses of the data at various concentrations of ACP. The results are the average of three determinations performed in duplicate. The $\mathrm{Km}$ for 7352, a low oil content cultivar, was $1.64 \pm 0.23 \mu \mathrm{M}$, and the Km for 7708, a high oil content cultivar, was $1.57 \pm 0.34 \mu \mathrm{M}$. These results indicated that the BnMCATs from different cultivars have a similar Km for ACP, suggesting that BnMCATs from different cultivars have the same affinity for ACP. 

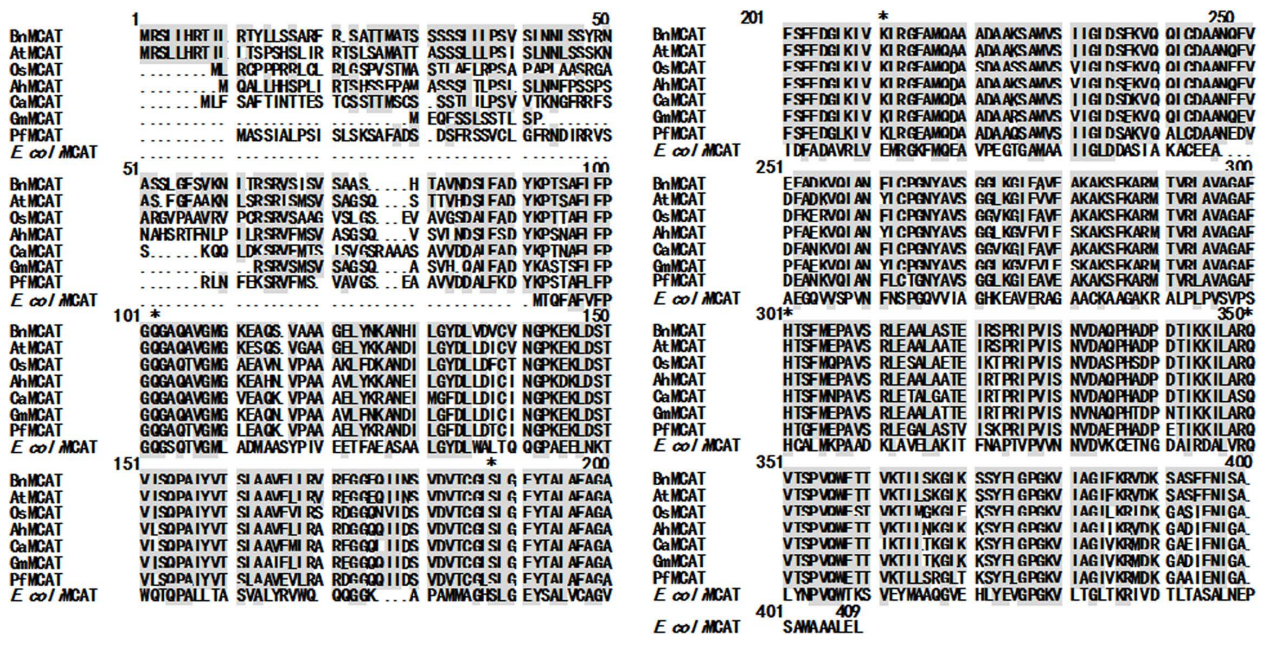

Figure 2. Multiple sequence alignment of MCAT. Protein sequences were aligned using the OMIGA software. Identity among BnMCAT and other MCATs are indicated by shaded squares. The conserved residues are shaded black and marked with an asterisk $(*)$. The GenBank accession numbers are as follows: AtMCAT, AAM14913; OsMCAT, ABF95452; AhMCAT, EU823322; CaMCAT, ACF17665; GmMCAT, ABB85235; PfMCAT, AAG43518; E. coli MCAT, 1MLA.

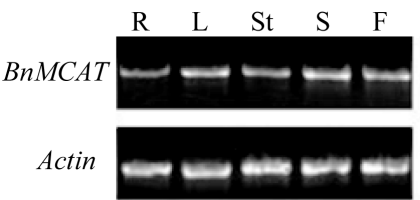

(a)

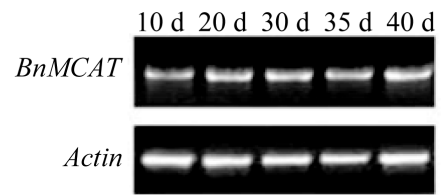

(b)

Figure 3. The expression of BnMCAT in rape. (a) The expression of BnMCAT in different tissues of rape; (b) The expression of BnMCAT in different seed stages after fertilization. Total RNA was extracted using Plant RNA Purification Reagent. First-strand cDNA was synthesized from $2 \mu \mathrm{g}$ of total RNA using Superscript II and Oligo(dT)18. A $0.5-\mu 1$ aliquot of the reverse transcription reaction was subjected to PCR using gene-specific primers BnMCAT 5' primer 1 (5' TTGTTCGCCGACTACAAACCC 3') and BnMCAT 3' primer 1 (5' GATGTTTTCGACGCTAGCA 3'). Actin was used as an internal control. R, root; L, leaf; St, stem; S, seed; F, flower.

Table 1. The enzymatic activity of recombinant BnMCAT.

\begin{tabular}{cc}
\hline & $\begin{array}{c}\text { Relative activity } \\
\left(\mathrm{nmol} \text { malonyl-ACP } \cdot \mathrm{mg}^{-1} \cdot \mathrm{min}^{-1}\right)\end{array}$ \\
\hline BnMCAT7352 & $42.41 \pm 0.56$ \\
BnMCAT7353 & $40.23 \pm 0.23$ \\
BnMCAT7699 & $42.21 \pm 0.60$ \\
\hline
\end{tabular}

Data are means of three biological replicates.

\subsection{Enzymatic Activity of BnMCAT in Rapeseed}

As an important enzyme in fatty acid biosynthesis, the enzymatic activity of MCAT in crude extracts isolated from rape leaves and different embryo stage were analyzed (Table 2); the results are the average of three determinations performed in duplicate. The values for BnMCAT activity in crude extracts were comparable to those found in spinach and sunflower crude extracts [7] [13] [14]. The relative activity of BnMCAT was similar at different embryo stages, with little variation, and a higher relative BnMCAT activity was found in embryos than leaves. The highest relative activity was found in 30-DAP (days after pollination) embryos, the walking stick stage. It was previously shown that abundant oil bodies are deposited in 30-DAP embryos [15], and the deposition of oil bodies requires a large quantity of fatty acid-comprised lipids. Therefore, oil deposition might pro- 


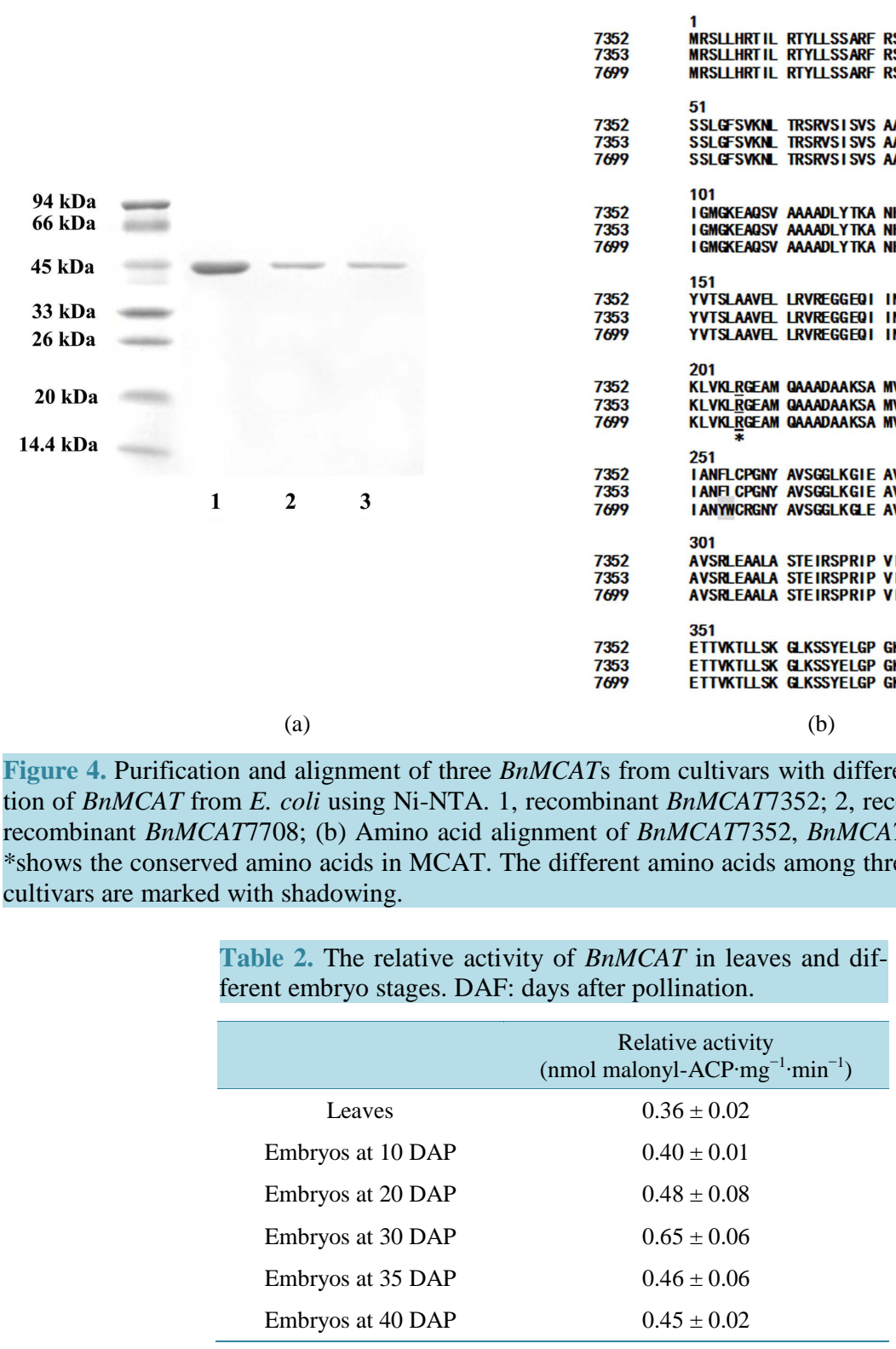

Data are means of three biological replicates.

mote fatty acid biosynthesis in embryos, and the activity of MCAT, as the most important enzyme in fatty acid biosynthesis, may be enhanced to meet this demand.

\subsection{Effect of $\mathrm{pH}$ and Temperature on the Relative Activity of BnMCAT in Rapeseed}

Enzymatic activity is affected by many factors, such as pH, temperature, and inhibitors [16]. Accordingly, we analyzed the effect of $\mathrm{pH}$ and temperature on the relative activity of BnMCAT to identify the optimal reaction conditions for this enzyme.

BnMCAT showed a typical bell-shaped $\mathrm{pH}$-dependence profile in the range of $\mathrm{pH} 5.0$ - 10.0. Maximum activity was observed at $\mathrm{pH} 8.0$, and this was reduced by approximately $30 \%$ maximum at $\mathrm{pH}$ values of 5.0 and 10.0 (Figure 5). This $\mathrm{pH}$ activity curve for BnMCAT is similar to that of GmMCAT, with a pH optima for GmMCAT at approximately 8.5; the GmMCAT curve also displayed a rather broad $\mathrm{pH}$ range for maximal activity between pH 7.5 and $\mathrm{pH} 8.5$ [14].

It was previously reported that the activity of GmMCAT was reduced by $50 \%$ when MCAT was exposed to 

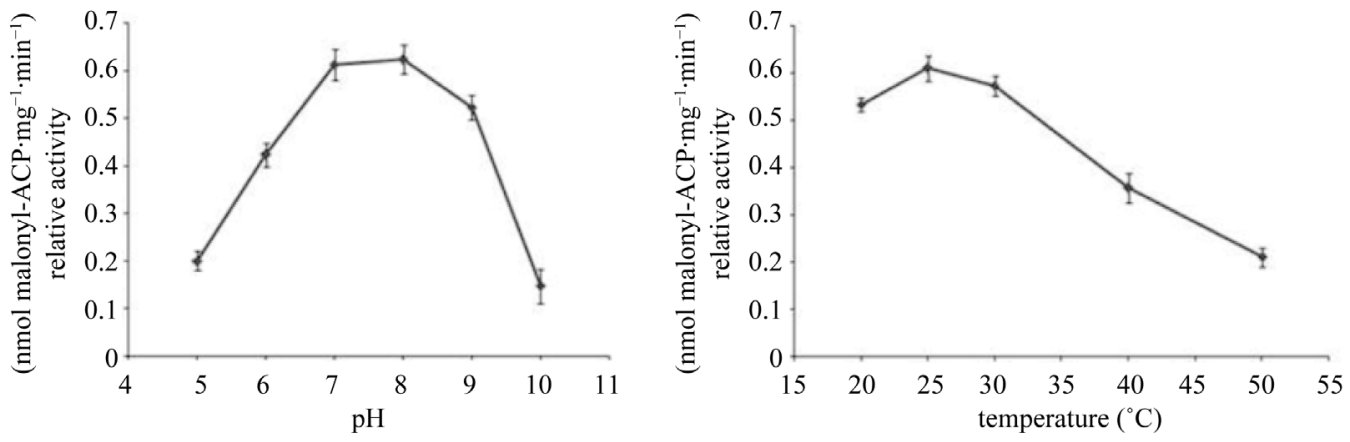

Figure 5. pH and temperature affect the relative activity of BnMCAT. Data are means of three biological replicates.

$50^{\circ} \mathrm{C}$ for approximately $5 \mathrm{~min}$, indicating that temperature can inhibit the relative activity of MCAT by destabilizing the protein [14]. BnMCAT also exhibited a temperature-dependent profile in the range of $20^{\circ} \mathrm{C}$ to $50^{\circ} \mathrm{C}$. BnMCAT showed maximum activity at $25^{\circ} \mathrm{C}$, and the relative activity showed a decreasing trend when the reaction temperature was increased: the relative activity was reduced to approximately $30 \%$ of the maximum at $50^{\circ} \mathrm{C}$ (Figure 5).

\subsection{Activity of MCAT from Cultivars with Different Oil Content}

Although rapeseed is one of the most important oil sources, it remains unknown whether the activities of fatty acid biosynthesis enzymes affect the rape oil content. Twelve cultivars with different oil content were chosen for an analysis of the relationship between the oil content and relative BnMCAT activity in immature seeds at 30 days after pollination (Figure 6(a)). The oil content from cultivar No. 1 (7352) to cultivar No. 12 (7708) increased gradually from $36.92 \%$ to $48 \%$ (Figure $6($ a), curve 1). However, the relative BnMCAT activity did not increase gradually and smoothly (Figure 6(a), curve 2). Indeed, the relative activity was at a very high level in some low oil content cultivars, such as in No. 1 (7352) and No. 3 (7351), whereas the relative activity was low in some high oil content cultivars, such as No. 8 (7353) and No. 10 (7699). Although it appears that there is no relationship between the oil content and relative activity, the increasing tendency of the relative activity in the 12 cultivars with the oil content suggests that the relative activity of BnMCAT might be related to the oil content in B. napus (Figure 6(a), curve 3).

Because MCAT is an important enzyme in fatty acids biosynthesis, the expression level might be related to its activity, thereby affecting the oil content. Therefore, the expression level of the BnMCAT gene in the different cultivars was analyzed (Figure 6(b)). Total RNA was isolated from immature seeds at after 30 days pollination, and the RT-PCR results showed that the expression level of BnMCAT in the low oil content cultivars was lower than that in the moderate and high oil content cultivars. However, this pattern did not fit for the middle and high oil content cultivars because the expression level of BnMCAT in the middle oil content cultivar was higher than in the high oil content cultivar (Figure 6(b)). Comparing this result with the relative activity in the different cultivars, no relationship was found for the relative activity of BnMCAT and the expression level of BnMCAT. Indeed, a high expression level did not indicate a high relative activity because the relative activity of BnMCAT was affected by several factors, such as $\mathrm{pH}$ and temperature (Figure 5), and other, unknown factors. These results demonstrated that the expression level of BnMCAT did not determine the relative activity of BnMCAT and the rapeseed oil content.

As the genetic background is one of the most important factors affecting the oil content, the genetic relationship among the 12 cultivars was analyzed (Figure 6(c), with the oil content and relative BnMCAT activity shown in parentheses). The oil content and relative BnMCAT activity showed a similar trend among cultivars with a close genetic relationship, such as 7350 and 7699, though 7699 cultivar had a higher oil content and relative activity than 7350. Although the 7351 and 7352 cultivars also displayed this pattern, a very high relative BnMCAT activity was found in these cultivars with a very low oil content, suggesting that there are internal factors determining the oil content and that MCAT activity is only one of them. All these results further demonstrated that the relative activity of BnMCAT played an important role in determining the oil content of B. napus, particularly in those cultivars with a close genetic relationship. 
(a)

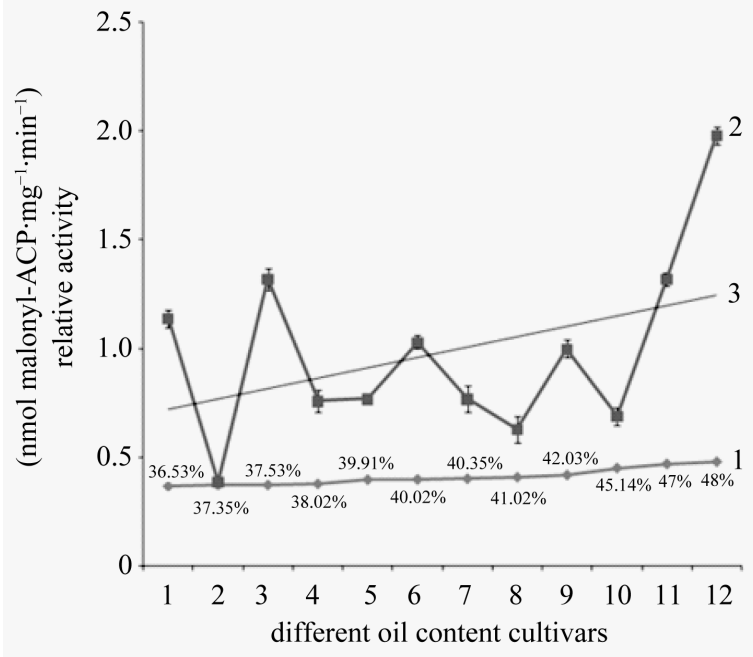

(b)

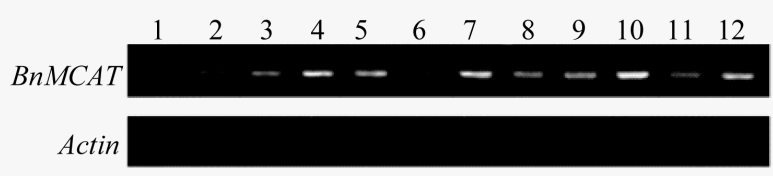

(c)

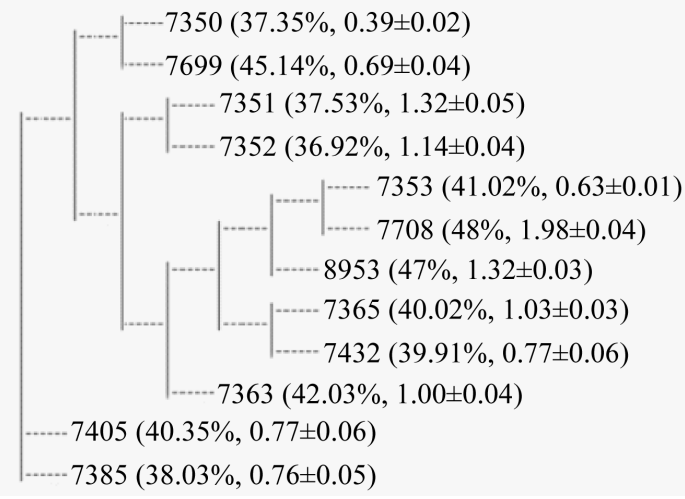

Figure 6. The expression and activity analysis of BnMCAT in different oil content cultivars. (a) The relationship between oil content and relative BnMCAT activity. Curve 1: the oil content of the 12 cultivars. The oil content of each cultivar is shown beside the curve point. Curve 2: the relative activity of BnMCAT in 12 cultivars. Curve 3: the trend line of the relative activity of BnMCAT in 12 cultivars. The figures on the abscissa axis show the 12 cultivars with different oil content. 1, 7352; 2, 7350; 3, 7351; 4, 7385; 5, 7432; 6, 7365; 7, 7405; 8, 7353; 9, 7363; 10, 7699; 11, 8953; 12, 7708; (b) The expression of BnMCAT in immature seeds at 30 days after pollination in 12 cultivars; (c) The genetic relationship among the 12 cultivars. Data are means of three biological replicates.

\section{Discussion and Conclusion}

\subsection{Malonyl-CoA:ACP Transacylase (MCAT) in B. napus Is the Most Important Enzyme in Fatty Acid Synthesis}

MCAT has received much attention as a possible antibiotic target because FAS II differs from FAS I in mammals, and great progress has been made over the last decade with regard to MCAT in pathogenic bacteria. Analyses of the crystal structures of MCAT from Escherichia coli [17], Streptomyces coelicolor [3], H. pylori [18], M. tuberculosis [19], Staphylococcus aureus [20], Streptococcus mutans strain UA159 [21], and Xanthomonas oryzae pv. Oryzae [22] have suggested that MCAT is one of the most promising targets for antibacterial agent discovery. Furthermore, it was reported that MCAT is the major target of isoniazid in tuberculosis treatment [23]. Regardless, the important roles of MCAT in plants have not been described to date.

However, plant MCAT activity has been verified through direct experimental evidence in vitro [24], and ACP has also been shown to have transacylating activity both in an FAS system and polyketide synthesis system [2], [25]-[27]. Additionally, bacteria with a temperature-sensitive variant of MCAT may also be rescued by ACP [26]. As these results imply that MCAT might not be necessary for the FAS and polyketide synthesis systems, it is important and necessary to analyze the activity and functions of MCAT in plants to clarify the status of MCAT in these systems. This dispute might be resolved based on an analysis of TcmM, an ACP in S. glaucescens, whereby a rigorous set of experiments showed that the self-malonylation of ACP was an artifact of the expression and purification protocols [28]. Further evidence is based on a genetic analysis in which the genetic inactivation of the MCAT gene has been shown to be lethal in all major pathogens investigated [29]. Such plants as in Brassica napus, Arachis hypogaea, and Arabidopsis have only one MCAT gene [5] [19]. In Arabidopsis, no T-DNA insertional mutant for AtMCAT was found because the deletion of MCAT is lethal for plant growth and development, and plants harboring an RNAi targeting AtMCAT showed less flowers and seeds and a dwarf phenotype (data not shown). These results demonstrate that MCAT cannot be complemented by ACP or other 
enzymes in plants.

\subsection{The Relative Activity of Malonyl-CoA:ACP Transacylase (MCAT) Plays an Important Role in Determining the Oil Content in Rapeseed}

It was previously shown that the over-expression of E. coli MCAT in rape and tobacco could not enhance the fatty acid and lipid content in seeds [12]. Based on this result, researchers maintained that MCAT does not catalyze the rate-limiting step in plant fatty acid biosynthesis and disregarded the functional analysis of plant MCAT. In contrast, Jeon et al. [30] and Zhang et al. [31] reported that the over-expression of E. coli MCAT can improve fatty acid production in E. coli, suggesting that MCAT is the most important enzyme in fatty acid biosynthesis and can affect the overall synthesis.

Our results showed an increasing tendency of the relative activity of BnMCAT in 12 cultivars with an increasing oil content; at the same time, the expression level of BnMCAT in these cultivars showed no relationship with the oil content and relative activity. It was reported that, when the level of activated MCAT was increased in a reconstituted spinach FAS system, a noticeable increase in $\left[{ }^{14} \mathrm{C}\right]$ malonyl-CoA incorporation and stearic acid formation was observed [24]. Thus, the enzymatic activity of MCAT is the most important factor determining fatty acid production and oil content. The over-expression of E. coli MCAT in E. coli might result in a high relative activity, thus improving fatty acid production [30] [31]. This hypothesis may also be used to explain why E. coli MCAT could not enhance the quantity of fatty acids when over-expressed in tobacco and rape, as E. coli MCAT may have a low enzymatic activity in transgenic plants [12].

Enzyme activity is affected by environmental conditions, and altering the environmental conditions can alter the reaction rate of the enzyme. $\mathrm{pH}$ and temperature are the main factors affecting enzymatic activity [16], and our results showed that $B n M C A T$ has maximum activity at $25^{\circ} \mathrm{C}$ and $\mathrm{pH} 8.0$, with the relative activity decreasing when the $\mathrm{pH}$ and temperature were changed. Furthermore, the BnMCAT amino acid sequence in different cultivars showed a very low diversity. Although the recombinant proteins from the different cultivars showed similar activity in vitro (Figure 4), the native BnMCAT enzymes had different activity (Figure 6(a)). These results indicate that there are unknown factors in rapeseed plants that influence the relative activity of BnMCAT.

Our results may explain why the same cultivars of rape have different sees oil content when planted in different locations or in different years, as environmental conditions affect the activities of BnMCAT and the other enzymes involved in fatty acid synthesis and oil accumulation. Our results suggest a new method to improve the oil content in rapeseed by increasing the relative activity of the main enzymes in fatty acid and oil syntheses.

Biotechnology-based approaches are important methods to enhance the oil content in rape seed. Our results showed that BnMCAT is an important enzyme in the FAS system and that BnMCAT might be a new target enzyme in future crop improvement through genetic engineering.

\section{Highlights}

1) In this paper, a full-length genomic DNA of BnMCAT was cloned and analyzed. BnMCAT showed high identity to AtMCAT and other plant MCATs in both DNA and amino acid sequences.

2) Purified recombinant BnMCAT exhibited transacylase activity. BnMCAT in crude extracts from different parts of Brassica napus plants had different relative activities, and a higher BnMCAT activity was found in the crude extract from embryos.

3) BnMCAT in crude extracts from cultivars with different oil content showed different relative activities, and a higher relative BnMCAT activity might be an essential condition for a high oil content in rapeseed.

Thus, as the most important enzyme in fatty acid synthesis, BnMCAT might be used to enhance oil contents through genetic engineering.

\section{Acknowledgements}

We thank Professor Jianru Zuo from the Institute of Genetics and Developmental Biology, the Chinese Academy of Sciences, for his helpful suggestion and comments with regard to this investigation. Professor Rongzhan Guan from Nanjing Agricultural University kindly provided the B. napus materials. This work was supported by the National Basic Research Program of China (Grant No. 2006CB101601), the Hubei Province Science Foundation (Grant No. 2011CDB06909), and the National Natural Science Foundation of China (Grant No. 


\section{References}

[1] White, S.W., Zheng, J., Zhang, Y.M. and Rock, C.O. (2005) The Structural Biology of Type II Fatty Acid Biosynthesis. Annual Review of Biochemistry, 74, 791-831.http://dx.doi.org/10.1146/annurev.biochem.74.082803.133524

[2] Arthur, C.J., Szafranska, A., Evans, S.E., Findlow, S.C., Burston, S.G., Owen, P., Clark-Lewis, I., Simpson, T.J., Crosby, J. and Crump, M. (2005) Self-Malonylation Is an Intrinsic Property of a Chemically Synthesized Type II Polyketide Synthase Acyl Carrier Protein. Biochemisty, 44, 15414-15421. http://dx.doi.org/10.1021/bi051499i

[3] Keatinge-Clay, A.T., Shelat, A.A., Savage, D.F., Tsai, S.C., Miercke, L.J., O’Connell, J.D., Khosla, C. and Stroud, R.M. (2003) Catalysis, Specificity, and ACP Docking Site of Streptomyces Coelicolor Malonyl-CoA: ACP Transacylase. Structure, 11, 147-154. http://dx.doi.org/10.1016/S0969-2126(03)00004-2

[4] Summers, R.G., Ali, A., Shen, B., Wessel, W.A. and Hutchinson, C.R. (1995) Malonyl-Coenzyme A: Acyl Carrier Protein Acyltransferase of Streptomyces glaucescens: A Possible Link between Fatty Acid and Polyketide Biosynthesis. Biochemistry, 34, 9389-9402. http://dx.doi.org/10.1021/bi00029a015

[5] Simon, J.W. and Slabas, A.R. (1998) cDNA Cloning of Brassica Napus Malonyl-CoA:ACP Transacylase (MCAT) (fab D) and Complementation of an E. coli MCAT Mutant. FEBS Letters, 435, 204-206. http://dx.doi.org/10.1016/S0014-5793(98)01055-2

[6] Hajduch, M., Casteel, J.E., Hurrelmeyer, K.E., Song, A., Agrawal, G.K. and Thelen, J.J. (2006) Proteomic Analysis of Seed Filling in Brassica napus. Developmental Characterization of Metabolic Isozymes Using High-Resolution TwoDimensional Gel Electrophoresis. Plant Physiology, 141, 32-46. http://dx.doi.org/10.1104/pp.105.075390

[7] Martínez-Force, E.,Álvarez-Ortega, R. and Garcés, R. (1999) Enzymatic Characterisation of High-Palmitic Acid Sunflower (Helianthus annuus L.) Mutants. Planta, 207, 533-538. http://dx.doi.org/10.1007/s004250050514

[8] Kremer, L., Nampoothiri, K.M., Lesjean, S., Dover, L.G., Graham, S., Betts, J., Brennan, P.J., Minnikin, D.E., Locht, C. and Besra, G.S. (2001) Biochemical Characterization of Acyl Carrier Protein (AcpM) and Malonyl-CoA: AcpM Transacylase (mtFabD), Two Major Components of Mycobacterium tuberculosis Fatty Acid Synthase II. The Journal of Biological Chemistry, 276, 27967-27974. http://dx.doi.org/10.1074/jbc.M103687200

[9] Bradford, M.M. (1976) A Rapid and Sensitive Method for the Quantitative of Microgram Quantities of Protein Utilizing the Principle of Protein-Dye Binding. Analytical Biochemistry, 72, 248-254. http://dx.doi.org/10.1016/0003-2697(76)90527-3

[10] Serre, L., Verbree, E.C., Dauter, Z., Stuije, A.R. and Derewenda, Z.S. (1995) The Escherichia coli Malonyl-CoA: Acyl Carrier Protein Transacylase at 1.5-A Resolution. Crystal Structure of a Fatty Acid Synthase Component. The Journal of Biological Chemistry, 270, 12961-12964. http://dx.doi.org/10.1074/jbc.270.22.12961

[11] Harwood, J.L. (1996) Recent Advances in the Biosynthesis of Plant Fatty Acids. Biochimica et Bioohysica Acta, 130, 7-56. http://dx.doi.org/10.1016/0005-2760(95)00242-1

[12] Verwoert, I.I., van der Linden, K.H., Nijkamp, H.J. and Stuitje, A.R. (1994) Developmental Specific Expression and Organelle Targeting of the Escherichia coli fabD Gene, Encoding Malonyl Coenzyme A-Acyl Carrier Protein Transacylase in Transgenic Rape and Tobacco Seeds. Plant Molecular Biology, 26, 189-202. http://dx.doi.org/10.1007/BF00039531

[13] Stapleton, S.R. and Jaworski, J.G. (1984) Characterization and Purfication of Malonyl-Coenzyme A: [Acyl-carrierprotein] Transacylases from Spinach and Anabaena variabilis. Biochimica et Biophysica Acta, 794, 240-248. http://dx.doi.org/10.1016/0005-2760(84)90151-6

[14] Guerra, D.J. and Ohlrogge, J.B. (1986) Partial Purification and Characterization of Two Forms of Malonyl-Coenzyme A: Acyl Carrier Proteintransacylase from Soybean Leaf Tissue. Archives of Biochemistry and Biophysics, 246, $274-285$. http://dx.doi.org/10.1016/0003-9861(86)90473-X

[15] He, Y.-Q. and Wu, Y. (2009) Oil Body Biogenesis during Brassica napus Embryogenesis. Journal of Integrative Plant Biology, 51, 792-799. http://dx.doi.org/10.1111/j.1744-7909.2009.00851.x

[16] Nelson, D.L. and Cox, M.M. (2008) Principles of Biochemistry. 5th Edition, W. H. Freeman and Company, New York.

[17] Oefner, C., Schulz, H., D’Arcy, A. and Dale, G.E. (2006) Mapping the Active Site of Escherichia coli MalonylCoA-acyl Carrier Protein Transacylase (FabD) by Protein Crystallography. Acta Crystallographica Section D: Biological Crystallography, 62, 613-618. http://dx.doi.org/10.1107/S0907444906009474

[18] Zhang, L., Liu, W., Xiao, J., Hu, T., Chen, J., Chen, K., Jiang, H. and Shen, X. (2007) Malonyl-CoA:Acyl Carrier Protein Transacylase from Helicobacter pylori: Crystal Structure and Its Interaction with Acyl Carrier Protein. Protein Science, 16, 1184-1192. http://dx.doi.org/10.1110/ps.072757307

[19] Li, Z., Huang, Y., Ge, J., Fan, H., Zhou, X., Li, S., Bartlam, M., Wang, H. and Rao, Z. (2007) The Crystal Structure of 
MCAT from Mycobacterium tuberculosis Reveals Three New Catalytic Models. Journal of Molecular Biology, 371, 1075-1083. http://dx.doi.org/10.1016/j.jmb.2007.06.004

[20] Hong, S.K., Kim, K.H. and Kim, E.E. (2010) Cloning, Purification, Crystallization and Preliminary X-Ray Crystallographic Analysis of MCAT from Staphylococcus Aureus. Acta Crystallographica Section F: Structural Biology and Crystallization Communications, 66, 20-22. http://dx.doi.org/10.1107/S1744309109045989

[21] Kim, T.O., Im, D.W., Jung, H.J., Kwon, S.J. and Heo, Y.S. (2012) Purification, Crystallization and Preliminary X-Ray Diffraction Analysis of Enoyl-Acyl Carrier Protein Reductase (FabK) from Streptococcus mutans Strain UA159. Acta Crystallographica, 68, 292-294. http://dx.doi.org/10.1107/S1744309112000115

[22] Natarajan, S., Kim, J.K., Jung, T.K., Doan, T.T., Ngo, H.P., Hong, M.K., Kim, S., Tan, V.P., Ahn, S.J., Lee, S.H., Han, Y., Ahn, Y.J. and Kang, L.W. (2012) Crystal Structure Of Malonyl CoA-Acyl Carrier Protein Transacylase from Xanthomanous oryzae pv. oryzae and Its Proposed Binding with ACP. Molecules and Cells, 33, 19-25. http://dx.doi.org/10.1007/s10059-012-2155-y

[23] Baldock, C., Rafferty, J.B., Sedelnikova, S.E., Baker, P.J., Stuitje, A.R., Slabas, A.R., Hawkes, T.R. and Rice, D.W. (1996) A Mechanism of Drug Action Revealed by Structural Studies of Enoyl Reductase. Science, 274, 2108-2110. http://dx.doi.org/10.1126/science.274.5295.2107

[24] Shimakata, T. and Stumpf, P.K. (1983) The Purification and Function of Acetyl Coenzyme A: Acyl Carrier Protein Transacylase. The Journal of Biological Chemistry, 258, 3592-3598. http://www.jbc.org/content/258/6/3592.long

[25] Arthur, C.J., Szafranska, A.E., Long, J., Mills, J., Cox, R.J., Findlow, S.C., Simpson, T.J., Crump, M.P. and Crosby, J. (2006) The Malonyl Transferase Activity of Type II Polyketide Synthase Acyl Carrier Proteins. Chemistry \& Biology, 13, 587-596. http://dx.doi.org/10.1016/j.chembiol.2006.03.010

[26] Misra, A., Sharma, S.K., Surolia, N. and Surolia, A. (2007) Self-Acylation Properties of Type II Fatty Acid Biosynthesis Acyl Carrier Protein. Chemistry \& Biology, 14, 775-783. http://dx.doi.org/10.1016/j.chembiol.2007.05.013

[27] Misra, A., Surolia, N. and Surolia, A. (2009) Catalysis and Mechanism of Malonyl Transferase Activity in Type II Fatty Acid Biosynthesis Acyl Carrier Proteins. Molecular BioSystems, 5, 651-659. http://dx.doi.org/10.1039/b820420a

[28] Florova, G., Kazanina, G. and Reynolds, K.A. (2002) Enzymes Involved in Fatty Acid and Polyketide Biosynthesis in Streptomyces glaucescens: Role of FabH and FabD and Their Acyl Carrier Protein Specificity. Biochemistry, 41, 10462-10471. http://dx.doi.org/10.1021/bi0258804

[29] Molnos, J., Gardiner, R., Dale, G.E. and Lange, R. (2003) A Continuous Coupled Enzyme Assay for Bacterial Malonyl-CoA: Acyl Carrier Protein Transacylase (FabD). Analytical Biochemistry, 319, 171-176. http://dx.doi.org/10.1016/S0003-2697(03)00327-0

[30] Jeon, E., Lee, S., Won, J.I., Han, S.O., Kim, J. and Lee, J. (2011) Development of Escherichia coli MG1655 Strains to Produce Long Chain Fatty Acids by Engineering Fatty Acid Synthesis (FAS) Metabolism. Enzyme and Microbial Technology, 49, 44-51. http://dx.doi.org/10.1016/j.enzmictec.2011.04.001

[31] Zhang, X., Agrawal, A. and San, K.Y. (2012) Improving Fatty Acid Production in Escherichia coli through the Overexpression of Malony CoA-Acyl Carrier Protein Transacylase. Biotechnology Progress, 28, 60-65. http://dx.doi.org/10.1002/btpr.716 
Scientific Research Publishing (SCIRP) is one of the largest Open Access journal publishers. It is currently publishing more than 200 open access, online, peer-reviewed journals covering a wide range of academic disciplines. SCIRP serves the worldwide academic communities and contributes to the progress and application of science with its publication.

Other selected journals from SCIRP are listed as below. Submit your manuscript to us via either submit@scirp.org or Online Submission Portal.
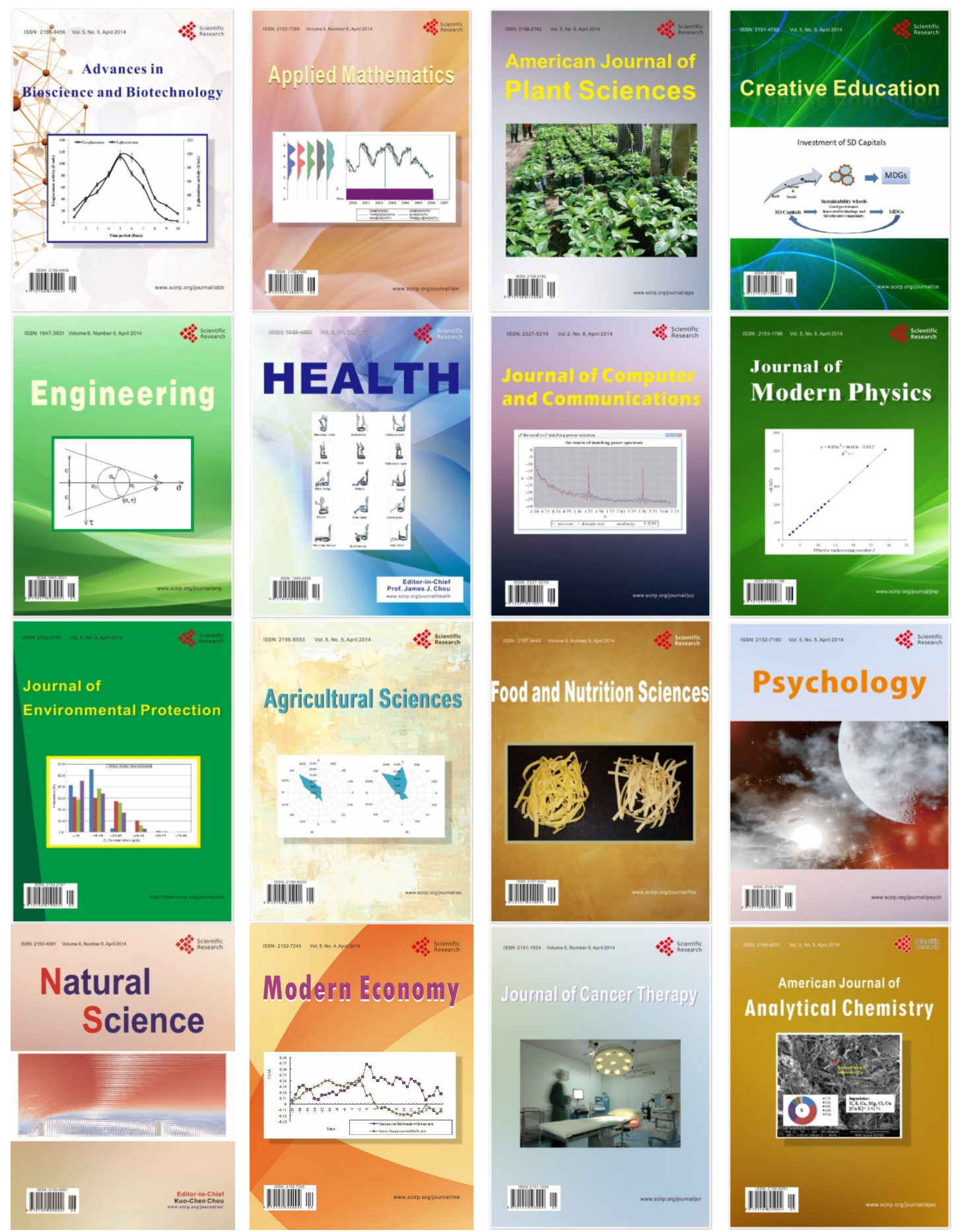